\title{
Business intelligence and predictive indicators for the performance-driven organization - Interview with Laura Patterson of VisionEdge Marketing
}

\begin{abstract}
Laura Patterson
has worked for both large public companies such as State Farm and Motorola, as well as for startups. Her marketing and sales career spans nearly 30 years. She often says that in her career she's only worked for two types of people, engineers and accountants, both of whom care deeply about data and numbers, and so her passion for metrics was really based on career survival. In 1999, she co-founded VisionEdge Marketing (www.visionedgemarketing.com), a data-driven, metrics-based strategic and product marketing company that specializes in improving marketing performance and helping organizations create a competitive advantage designed to attract, secure and retain profitable customers. Author of dozens of published marketing and branding articles and the books Gone Fishin', Measure What Matters and the soon to be published Metrics in Action: Creating PerformanceDriven Organizations, Laura has served on several nonprofit boards, has provided programming for professional organizations such as the ANA and BMA and has served as a guest lecturer at various universities, including the University of Texas at Austin, Purdue University, Tuck's Business School at Dartmouth College and Stanford University. Laura earned her B.A. from Truman State University and her Master's at the University of South Florida.
\end{abstract}

Keywords: marketing analytics, dashboard, marketing metrics, predictive analytics, marketing models, branding

Abstract Michael Moon interviews Laura Patterson, Principal of VisionEdge Marketing, on performance metrics: how predictive models and key performance indicators (metrics embedded with performance targets, projected values, share of wallet, etc) can enable an enterprise to calculate the ROI of their marketing efforts. Journal of Digital Asset Management (2008) 4, 213-224. doi:10.1057/dam.2008.25

Laura Patterson VisionEdge Marketing, PO Box 342546 , Austin TX 78734, USA Tel: 5126818800 E-mail: laurap@ visionedgemarketing.com
MM: We're here with Laura Patterson President of VisionEdge Marketing. Please give us a little bit of your professional background. LP: Michael, I began my career nearly 30 years ago in sales and marketing. I started in the financial services and healthcare industries. This was before computing and mobile communications technology was such a primary part of the American economy.

Early in my career, I ended up with an opportunity to join a company that had a medical billing application that ran on a Wang 2200. This opportunity served as my entree into the world of technology. From there, I went to work for Motorola Semiconductors for 14 years, and then back into financial services and enterprise software. Then in 1999, I co-founded VisionEdge Marketing (VEM).

The thing I often say about my career is that I've had the privilege of primarily working for two kinds of people in my career - accountants and engineers. Their focus on the numbers had a huge impact and has played out in the work we do at VEM.

MM: Great. Why don't we talk a little bit about your current company, what it does, and the clients that it serves?

LP: Wonderful. Appreciate you asking what we do. When we began in 1999, I like to say we were a little bit ahead of our time. As a datadriven company, we were focused on helping companies make fact-based decisions and creating performance-driven marketing organizations. We believe in using data and analytics to help marketing organizations be more successful, more effective and efficient. We work with all kinds of companies, in every size and in every industry. Some of the customer's we've served include Adobe, ClearOrbit, Briggs \& Stratton, Kronos, Motion Computing, Skybitz, Tektronix and Zebra Technology. 
In 1999, the B2B world really wasn't quite ready to talk about marketing accountability and marketing metrics. We had a train, but there was no station, and there really weren't any people ready to ride. You may recall, in 2001, some of the first research was being done in the area of marketing accountability, and some of the first study results were coming out. By the early 2000s, people were starting to give the issue more attention. So it's been exciting to be at the beginning and the forefront.

MM: Cool.

What are some of the historical trends or history of metrics and ways of measuring accountability? Perhaps just in general - and then in particular, within marketing organizations.

LP: Well, measurement and accountability have actually been around a long time. Companies that have been manufacturing- or engineeringoriented have leveraged performance improvement techniques such as Six Sigma. Six Sigma has provided a good foundation and enables organizations to measure their performance and contributions in a variety of ways - both in terms of effectiveness and efficiency. And sales, of course, has always had performance measures, as has customer service. The Balanced Scorecard also provided a boost in terms of performance measurement.

The real gap in $\mathrm{B} 2 \mathrm{~B}$ has been marketing measurement. Of course, we have always been able to count things such as response rates, press hits and, more recently, open and click-through rates, but we weren't measuring in terms of our impact on and value to the business in quantifiable terms. And while marketing embraced counting things, we have a tendency to avoid setting performance targets and forecasting results. Michael, I know you've had a long career, as well - so perhaps that will all ring true with you.

MM: Laura, this gets to an underlying issue. Oftentimes it's a source of confusion - the difference between measuring and counting. Specifically, how you compare and contrast quantification - and how you measure quality.

Could you clarify the notion of quantification - both qualitative and quantitative?

LP: Yes. That's an excellent observation. First, quantitative metrics are statistical in nature and qualitative measures are based on judgment and perception. Both can be quantified, or as we like to say "numberfied." And both can be valuable and categorized in what we call the "metrics continuum." If you can envision the continuum from left to right at the bottom, metrics at the bottom far left we refer to as activity-based metrics. At the far upper right, we call those predictive metrics, with operational, outcomebased and leading indicators in between. This framework works very well when you start thinking about the three common levels of a dashboard: strategic, operational and tactical. Ideally metrics cascade down from the top to the bottom. (Figure 1)

Counting often occurs the most when we talk about activity-based metrics, because this is one of the easiest things for marketing to measure; things like we've already discussed, things like how many people attended an event, or downloaded a document or participated in a demo. But these are not adequate measures in terms of demonstrating value, nor do they give marketing insight into what is and isn't working. Metrics and measurement give marketing influence and power; armed with data and analytics we can frame strategic decisions. So performance measurement and management is about more than accountability and measuring progress, it is also about optimizing performance and empowerment. It's about being able to say, "Here's what we're going to do, here's the business impact it's going to have and here's how we're going to measure success, impact and value." Does that make sense?

MM: Yes.

LP: And the timing is right for marketers to take the initiative. The current economic and competitive environment and the limitation of resources are all contributing to this need for and emphasis on marketing people to create performance-driven organizations. Organizations that are looking at how they are contributing to the value of the company, impacting the business, measuring that contribution and helping the organization achieve a better return on the dollars invested. With metrics, marketing can gain more control over its destiny and have more influence within the organization.

MM: One of the things that seems to have really changed the pressure upon marketing has been the web. More specifically, the data that web interactions produce. It's made the positive 


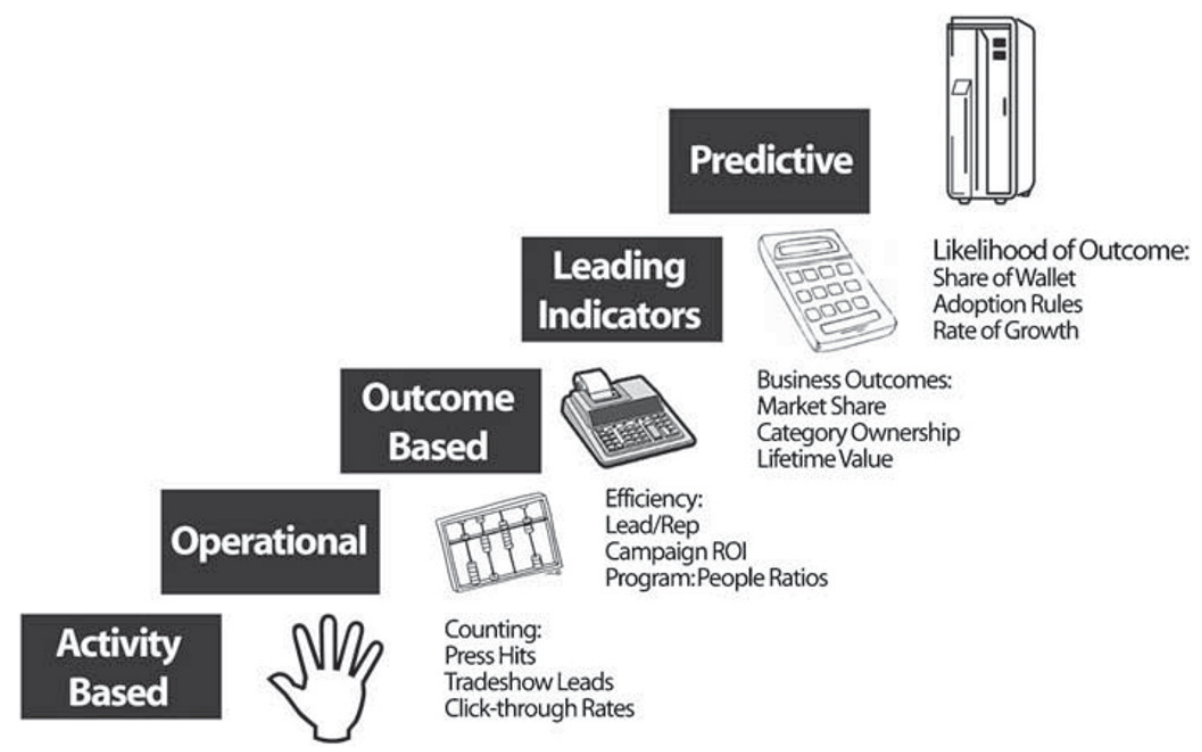

Figure 1: VisionEdge marketing metrics continuum

or negative effects of marketing much more transparent and self-evident. Seeing the correlation between, for example, a flight of ads and activity on the website.

Could you take us through, again, some of the other pressures that have compelled or induced marketers to get more accountable with respect to their spend?

LP: Michael, marketing is investing money on behalf of the company, and therefore we need to report on how we are using this money. Marketing performance measurement isn't just about spend; it's about making smarter decisions and communicating value. The exciting thing about the web was it made real-time analytics possible. Today, nearly every company can afford to add CRM, MRM, DAM and SFA capabilities that can help you track and monitor activities - things that didn't exist 10,15 or 20 years ago, and those that did were beyond the reach of most firms. The availability and affordability of better systems, tools and processes has made it possible for marketing to do a better job of measuring and tracking.

So while we might have been able to track our investments and results, in the past it was an arduous manual process. What we did in the past only allowed us to take a rear-view mirror approach. Today, things are more automated and we can be expected to think in terms of what the company is getting for the money that's being invested, not just with respect to spend, but in terms of being able to take a forward-looking view.

The systems and tools and processes that we can put into place today give us the ability to understand the relationship between programs and outcomes, to see patterns and trends, to measure progress in real or near-real time so we can make adjustments in mid-stream.

MM:Yes. Would it be fair to say that in terms of resources a marketer now is looking at reallocating or realigning using a more predictive model?

What are the kinds of things that predictive analysis really helps clarify other than, "We should do more of this or less of that?" LP: Yes, with tools, systems and rigorous data processes, a company can eventually create predictive models. Predictive models depend on accurate data, so quality data are essential. They also require data over time so patterns and trends can be used to do what if analysis and understand which initiatives and which combination of things will produce the desired result faster and more cost effectively. Predictive models help with better targeting - deciding which customers you should invest in.

While this may be highly desirable for all marketing organizations, many B2B companies are a long way from being able to create predictive models. They just don't have the data 
and so many firms are still in the "counting" phase. If a company can at least achieve outcome-based measurement, they've made huge progress in enabling themselves to demonstrate value and help facilitate better strategic decisions. MM: This reminds me of a paper that I read in MIT Sloan Management Review the spring of 2007. It's a paper written by Michael Hammer, entitled "The Seven Deadly Sins of Performance Measurement." He makes the case that it's really important to measure the right things, and it's really important to know what measuring the wrong things entails.

LP: I totally agree with that.

MM: What are some of the things that you found in your practice that one shouldn't attempt to measure? Either because the measuring of them doesn't produce any good information, or because it leads you down a rabbit hole, so to speak? What are some of the things that we shouldn't measure?

LP: This an insightful question because today it is possible to drown in a sea of metrics; our ability to measure has become almost infinite. I encourage our customers not to measure anything that isn't linked or aligned with a business outcome. On the flip side, marketers should measure everything that tells them whether they are producing the desired result. We recommend developing a basic results chain to help with this process - this provides a vehicle to demonstrate how activities/outputs are linked to and will lead to the achievement of each outcome. This way marketing knows where to focus and what to measure. (Figure 2) MM: Could you give us an example of some really crisp, well-formed performance objectives? LP: Sure. To help us form these, let's keep in mind marketing's three jobs - find, keep and grow the value of customers. So we can use these as the foundation to craft well-formed measurable marketing objectives. For example, if a company is entering a new segment and has an outcome to achieve some number of customers in this segment, a measurable performance objective for marketing may be "achieve some specified share of preference within X time period" within this segment. Another example might be related to increasing the value of a particular customer set by having them purchase a new product or additional products. You might have an objective around, "X number of customers in this set adopt the new product within some period following the product launch" or "increase the average number of products per customer in this set from $\mathrm{X}$ to $\mathrm{Y}$ by the end of the year." Does this help?

MM:Yes.

LP: So now we understand the outcome and have performance-based objectives - we can create key performance indicators (KPIS) essentially metrics embedded with a performance target, related to customer value, share of wallet, preference, etc. And we can see the connection between a marketing program around launching a new product and a change in customer value and a marketing program designed to change preference and revenue in a new market.

Is that an answer that you were looking for? MM: Perfect.

One of the things I recently ran across was a paper in Brandweek by the Miller and Heiman folks. They have written a fabulous book. It's kind of a classic in the field, called "The New Strategic Selling," by Robert Miller and Steven Heiman. Mr. Miller, I think, wrote a piece where he said, "You know, a sales funnel really has three parts to it." The first part is characterized by prospecting, the middle part by nurturing and the bottom half by closing.

He says that most organizations typically suffer some pretty vicious highs and lows in terms of monthly revenues. He says that structurally, that's a function of spending too much time in the closing part of the funnel,

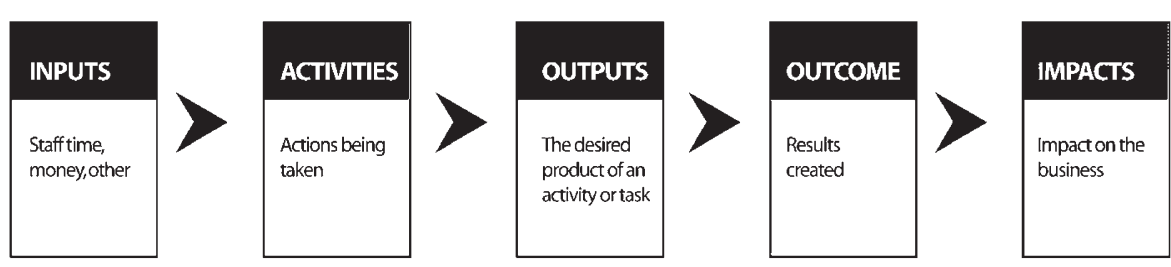

Figure 2: Basic results chain 
and not enough time in the nurturing and prospecting part of the tunnel.

The way out is to spend more time in the prospecting part — incrementally — in that there's a certain number of prospects that once you get them into the funnel, they'll race right through the buying process and buy things.

So in the context of managing a sales funnel with three parts - prospecting, nurturing and closing - what are some of the things that you look for in a dashboard or metrics in terms of measuring the efficiency and effectiveness of moving business through the sales pipeline? LP: That's a good question. By the way - we actually don't call it a sales pipeline. Just to share a philosophy, we talk about it being a customerbuying pipeline. We prefer marketing and sales people to think about the process from the customers' point of view. If you talk about it as a customer-buying pipeline, marketing and sales own the whole pipeline together. When it's referred to as a sales pipeline, it almost suggests there's no role for marketing.

We actually think of it as a pipeline instead of a funnel, where a bunch of stuff goes up in the top and somehow a few things come down at the bottom. By laying it on its side we can illustrate a pipeline with opportunities coming into the pipe and then moving through the pipe based on the customer buying process. Each stage of their process can then be segmented by their behavior.

Opportunities are generated in a variety of ways: some from sales, some from customer referrals and many from marketing. So from "finding" a customer's perspective, marketing should be looking at how it contributes to the pipeline - both in terms of rate and cost, as well as conversion from one stage to the next.

Opportunities at some stages will require nurturing; this is particularly true in $\mathrm{B} 2 \mathrm{~B}$, where decisions generally involve several participants from the customers and a number of steps in the buying process. So it will be important for marketing to understand what touchpoints are required along each stage of the customer's buying in order to keep that customer engaged and help them determine whether or not they want to continue to move forward, hopefully with your company. This is why it is so important for marketing to build preference: even if you are new company where awareness is important, ultimately it comes down to preference. The goal really is, "How do we create more preference for our products/services in the market so we can be among the top choices?". That's part of the nurturing part. Right?

MM: Yes. You know, Laura - I really like how you reframed the sales funnel into a customerbuying pipeline.

LP: We think of marketing and sales as partners in a dance. They're two sides of the revenue coin. They therefore have to work together. The whole issue of the marketing sales divide is really about marketing and sales alignment. Sometimes if you just reframe, you begin to make the conversation move forward in a positive direction. We explore this idea and process in our book Gone Fishin' for Marketing and Sales Alignment, if you ever want to check that out, Michael.

MM: Fabulous. I really like how it also then removes an artificial barrier between sales and marketing. It says, "It's all about facilitating the buying process." Or starting and facilitating the buying process. We've been talking here in terms of generalities. Clearly there are marked differences between a consumer brand and a B2B brand.

LP: Yes.

MM: A consumer brand really usually has some sort of retail channel between it and the customer. It really wants to focus on things like brand awareness and brand interaction, and perhaps some coupons or loyalty marketing. The B2B organization really focuses on lead generation and some sort of buying interaction vis-à-vis an RFI or an RFP.

Could you take us through some of the additional refinements in terms of performancebased metrics or performance-based objectives as they relate to consumer or $\mathrm{B} 2 \mathrm{~B}$ marketing operations?

LP: I'm not so sure that there are usually differences in terms of measurements when it comes to B2C or B2B. Marketing has the same purpose. We are given money to invest on behalf of the company in both situations. The company wants marketing to get more of something, faster and less expensively. Otherwise they would just put more feet on the street. Ultimately, our job in marketing is to produce profitable customers - whether those are 
individuals who are buying in a grocery store or online, or making a considered enterprise software purchase.

MM: As we have worked with both consumers and B2B, we've found that there's actually a pretty significantly different set of operational capabilities for consumers. They tend to spend a lot more money on traditional measured media - advertising, broadcast and print. They tend to spend a lot more money on point of purchase packaging and store merchandising and so on.

LP: True, where they spend their money may be different, but what they're being asked to produce - profitable customers - isn't.

MM: Absolutely.

LP: As a result we recommend marketing focus on the outcome. When we look at performance measures, it's always in the context of outcomes. So nearly every company - whether they're $\mathrm{B} 2 \mathrm{~B}$ or $\mathrm{B} 2 \mathrm{C}$, depending on their stage - is looking at how to grow revenue, how to increase market share, how to retain more profitable customers and increase customer value - how to get those customers to buy more often and buy more of the same or more of other products.

Usually there's some set of business outcomes around those kinds of goals. Depending on the company and customer buying process, the marketing organization may deploy a different set of tactics. But each company is still going to have to be moving those dials at the top. So we try to get marketing organizations to think about the business needles at the top they have to move - instead of what you so often see of B2B marketing plans, which are often about a list of activities designed to generally leads, such as executing some number of tradeshows during the year or deploying some number of e-mail campaigns.

Marketing, whether $\mathrm{B} 2 \mathrm{C}$ or $\mathrm{B} 2 \mathrm{~B}$, needs to make the connection in terms that demonstrate value to and impact on the business, in terms of better and more opportunities, faster and more cost effectively, with higher conversion ratios, ultimately resulting in more profitable customers. MM: Sure.

I had a conversation with a firm over in Finland around their marketing metrics. They said, "We really break our metrics down into three basic categories. We have efficiency metrics - which are about cost, spend of budget, cycle time and that sort of stuff. We have effectiveness metrics - which are those things that you were talking about in terms of key performance indicators of sales. Brand preference, repurchase and things like leads to channel and stuff."

Then the third one was most interesting. That kind of gets us to your point around "predictive models." This particular firm was a telecommunications firm.

The third metric I found most fascinating they referred to it as "Business Impact Metrics." I asked them what they meant by that. They said, "Well, the telecommunications business falls into what we call a "fast-cycle" brand market. That's to say that if you stop advertising for a couple of weeks, you begin to notice almost immediately a softening of revenues.

Fast-cycle brand markets require constant advertising reinforcement. Largely, that reflects both the quick turn of inventory or consumer intention, or just the inherent intangibility of the product.

So I said, "What do you mean by business impact?" They said, "Well, think of it this way. If we drop 100,000 direct mail pieces into this particular market, and we get a 2 per cent response rate, that's 2,000 responses. Now if we have 2,000 responses, do we have enough people in the call center to handle 2,000 phone calls over this particular period of time? If not, then we should probably think about either putting more people on - calling more people in to work - or scaling back the mailings."

The second thing they said was, "Now, if we get a 40 per cent conversion rate for the people that call in - that's 800 hookups. Do we have enough trucks on the streets to facilitate 800 new hookups for this particular service? If not, should we schedule out the delivery of the direct mail, and therefore the load on the call center, and therefore the conversion hookup lead time?"

So it's a dynamic simulation. We're going to spend a considerable amount of money doing this particular campaign or effort - and as we go through the whole execution value chain, do we have any weak links that will compromise or undermine the value of the investment?

Can you take us perhaps to the next level either in terms of the efficiency, effectiveness or business impact along the lines of metrics? And the kinds of things that CMOs are looking for? 
LP: That's an interesting idea, and I love the example you just used. We do look at business impact a little bit differently.

When we talk about metrics and measuring marketing performance, we are looking at it from an outcome point of view. That is, what are the business outcomes? What are the ultimate needles marketing is executed to move? And how is marketing going to measure that it contributed or made those needles move, in terms of the actual movement - the value as well as the time - the efficiency of it, at what cost for what value?

There could be several needles. A company generally has several business outcomes that they're trying to accomplish. They might be around turning a product into an industry standard, or growing the value of a particular set of customers, or bringing a new product to marketing that is quickly adopted or penetrates a particular region or set of accounts. What marketing needs to understand is what needle it is expected to move. Once the organization understands this, then marketing knows what it needs to do to perform and what it needs to measure in terms of effectiveness, value and efficiency.

By understanding the outcome that marketing is expected to produce, the marketing organization is laser-beam focused on the right things, as opposed to putting together and measuring a bunch of stuff that may or may not even drive what the business is trying to achieve.

We just completed our seventh annual marketing performance survey. We took a look at the trends over the last four years. There are still really big challenges facing marketing. We are measuring or making good progress. The results suggest we're still not demonstrating value, not just ROI, but value to the business. Am I making sense, Michael?

MM: Sure.

LP: So while we are becoming very good at counting, we're not showing value. Something else that the study revealed is that our ability to measure is still perceived as being marginal elementary and not very sophisticated, compared to other parts of the organization such as sales, manufacturing, implementation or deployment.

We have the tendency in marketing to still focus on the short-term tactical things, like a campaign - and not on the strategic things.
That's another hurdle. So we need marketing to be a performance-driven organization that moves the company more strategically forward, as opposed to just executing on a particular tactical effort.

We also still have marketing organizations and marketing people who are struggling analytically. Creating a performance-driven organization depends on data and analytics, so marketers need to exercise their analytical muscle. If you look at the number or proportion of analytically oriented people in the market in organization, compared to the creative and communications people, it's not perceived as balanced. It's getting better, but it's still a problem.

The last thing that showed up is there is a gap still between "Talking the talk" and "Walking the walk." Marketing leadership is talking about measurement, but it still isn't a priority. The budgets and resources aren't being allocated. And many companies have no idea what the benchmarks are in their industry, or even broader terms for measuring marketing's contribution. They don't do audits. They don't invest in systems, processes or tools. So they really aren't putting the infrastructure in place to support performance measurement and management. These are things marketing needs to address if they want to create a performancedriven organization. Does that help?

MM: Sure.

It seems to me, Laura, that if I could summarize what you're saying: There's a fundamental mindset that does not seem aligned with the notion of performance-driven marketing. This mindset is - first of all marketing is creative. It's not procedural.

A lot that we do is inherently intangible and difficult to measure, anyway. Why do I want to start measuring things - especially things over which I have little or no direct control, and maybe only marginal influence?

It seems to me that a lot of marketers are very occupied in the day-to-day activities. They don't really think in terms of a broader system — nor in terms of holistic accountabilities to the business.

Is that a fair summarization? LP: I think so. We try to help companies think about five key areas: data, metrics and measurements, process, culture and skills, and capabilities. 
We find that a lot of companies still don't have data management systems in place that will really give them the information they're looking for. In fact, most of the time the marketing people have to go to other systems in the organization - such as the financial system or a customer service system to get the data. So, data are key areas.

When it comes to metrics, sometimes it's a matter of sitting down and having a conversation with the organization about, "How would you like us to measure our value?" I asked the marketing people, "Have you talked to your leadership team? The finance person? Your CEO? Your sales VP and so forth?" "Do you understand what role and impact they expect marketing to have?" Without these conversations, we are just making it up. This conversation needs to take place so they can design and select metrics that are really going to be driven by the questions that business users have.

Marketing needs measurement systems that they can consistently use. One of the things that we often ask people is "Have you defined your metrics? What's the calculation you have in place?" So that it's consistently used and applied every single time. As opposed to, "This month we're going to measure it this way, and next month we're going to measure it another way." That happens frequently. Or different organizations have the same idea for metrics, but they don't measure it in the same fashion.

Process. A lot of organizations haven't documented and/or operationalized their measurement processes. It's kind of ad hoc. They may have a way of creating a report, or even creating a graphical dashboard. But they don't really have a documented and proven process.

That kind of comes around to the last two, which we call "Living a measurement culture." Or, "Creating a culture of accountability." In a lot of organizations, there is a person or a team that is tasked with measuring marketing. Whether that's a marketing operations organization or an analytics person. It's their job. The rest of the organization is going about doing business as usual. When this is the case, this is a culture that sees measurement as separate from the rest of the team. Every marketer needs to understand the power of measuring performance. Does that make sense? MM:Yes.
Laura, it seems to me that - as you enumerated - where do you focus? Data, metrics, processes, people, or culture and skill sets? It seems to me that it still comes back to a particular management mindset. Somehow there's a conversation between the CEO and the $\mathrm{CMO}$ such that these conditions persist. LP:You are hitting the nail on the head. There needs to be executive-level championship or sponsorship for this kind of initiative. The CEO and CFO need to be a part of the process. MM: My question is, "Is that true?" Here's the reason I'm asking it. If I'm a CMO, the last thing I want to do is to look around for a stick for someone to beat me with.

So metrics, at the end of the day, are sticks. Right? Character sticks. But at the end of the day, they always come back as something I'm going to get beaten with.

I'm just a little perplexed as far as how a $\mathrm{CMO}$ would want to come up with operational performance metrics, only to have those used against them.

Really, if anything, I want to come up with metrics that I've gained and somehow pre-fed in advance - almost as the structure of how we count and quantify. So it shows that I'm meeting or exceeding my stated objectives. LP: Michael, we cannot be exempt from measuring; the rest of the organization technical support, customer service, engineering, manufacturing, sales, and so on are all being held accountable and have metrics. If we want a place at the table we need to be willing to put up the stakes. We need to be willing to put a stake in the ground and commit, just like sales or manufacturing, to performance targets. Secondly, metrics aren't just a stick. They're power. Data, analysis and proven performance give us power to influence strategic direction. MM: Laura, I agree with you completely. LP: The vice-president of sales — isn't he every month or every quarter making a commitment to the organization?

MM: Sure, he is. And he or she makes commitments both to understanding the reality of their existing pipeline, as well as to things that inevitably they will sandbag in the event that they need to pad this month or this quarter, or not.

LP: True. Then why should marketing do any less? Marketing should be willing to put a 
number on the table - whether it's sandbagged or not. We cannot just track the results after the fact and then arbitrarily declare success. At a minimum, we need to set a performance target and communicate how well we performed to this target.

MM: As many marketing executives have. That's my point.

LP: Yes. We appear to be in total agreement, Michael. So your next question to me is...? MM:You have a practice and a business. LP: Yes. And a passion.

MM: It really stands for bringing performancedriven marketing to organizations.

LP: Yes. And we have a book in the making, which we hope will be out by late summer, tentatively titled Metrics In Action: Creating a Performance-Driven Marketing Organization. In the book we're trying to give readers - from the $\mathrm{CEO}$, to the CMO, to anyone in marketing real ideas that they can put into practice.

MM:You do that as a function of saying, "Okay. Here are the metrics that really make a difference." Metrics in terms of quantification and quantification in terms of other intangibles. Here's how they correlate to business value either in terms of increased share of market, increased share of wallet, greater consideration in the buying decision process, etc. Those are all great.

In part of that, you're saying in order to really have those metrics become part of a culture, they need to be part of a larger system where you've got good data, you've got good processes, good automation underneath those processes and people who use them.

LP: That would be the ideal, although I'm not sure that perfect scenario exits in many places.

MM: No. I understand.

LP: Even without all of these in place right now, a marketing organization can begin to make progress. Depending on where they are, it can be a first small step related to setting performance targets for programs, or a bigger step related to creating measurable objectives tied to outcome. Of course, in each instance, they will still need to be able to collect and process data. So our passion is to help marketing measure and improve value and impact. This does require marketing to embrace data, because data are the foundation for creating metrics and metrics give us insights into performance.
MM: All right. So let's then talk about the areas within marketing operations that typically get excited about what you've just said. Who's typically the change advocate or champion for this new way of marketing?

LP: The CMO - because they recognize that they will gain more credibility and have a more strategic role in the company if they get involved.

By becoming a performance-driven organization and by working with the leadership team, marketing, especially B2B marketing, becomes something more than sales support. Most CMOs want to play a more strategic role and have a more influential voice - this takes measurement and accountability.

MM: Let's say that that CMO goes, "Laura this is really great. We really need to do this." Then what happens?

LP: Good question. Some companies are a little ahead of others and have a marketing operations director or VP or function. That often is a place where we might start working together. Not every company has that. It's a function that has been emerging over the last few years within many B2B companies. For companies that don't have this structure, common places might lie with the person responsible for segmentation, business intelligence analytics may be able to lead the effort - people typically with analytical responsibilities as opposed to the marketing communications person.

MM: If you could, would you draw a "goodbetter-best" three versions of the "ideal" operational executive, to really bring performancedriven marketing into their organization. And more specifically, what are the skills, aptitudes and perhaps career achievements that an executive has had minimally, better and/or best?

LP: Boy, that's a big question. I'll do my best. First, regardless of good, better and best, the operational executive needs to be analytically inclined and very comfortable with data. In addition, they should be process-oriented and believe in a disciplined systematic methodology. Does that make sense?

MM: So in terms of being data-inclined, not only are they comfortable with spreadsheets, but they also understand the underlying structure of databases, and how data are then transformed into information. And how certain kinds of information transformed well produce insights. 
LP: Yes, at minimum.

MM: The other thing you said was that they are process-oriented.

LP: Correct.

MM: Part of being process-oriented comes from the mindset, "I can manage people or I can manage processes," with the caveat that people are unmanageable.

LP: Really? How about this idea? "I can use data and process as a way to help improve performance, and make people feel better about their work."

MM: So strong data, strong processorientation....and what else?

LP: Those are two. And they are required at the minimum level. Now for "better." People in the better category are people who are change agents and can address change management. Performance measurement and management often has cultural implications. Those people who are "better" understand and know how and when to bring in new systems and put in the right infrastructure - the systems and tools - and then integrate these into the rest of the business.

These people should be able to start taking the insights revealed from the data and help the marketing organization communicate these in some fashion up, down and across. Whether that's with a dashboard of some kind or other vehicle, that would be "better." They would be bringing performance intelligence to the conversation. .

Then at the very top, the best can actually take the data and begin to build models, enabling the organization to ask, "What if? What if we did this? What's the likelihood of A occurring or B occurring?" That's a pretty high-level skill.

MM: That's what you mean by a change management or change facilitator?

LP: Yes.

MM: In terms of our good-better-best - at the "better" level is the marketing operations executive who will drive performance operations into marketing.

First of all, you'd mentioned that they have to facilitate change. Facilitating change - in my way of understanding it - really breaks down into a couple of things.

One is being able to conceive of a master project plan, and then break it down into smaller and smaller more easily executed chunks. Being able to find or source appropriate people for those smaller chunks - all the while kind of orchestrating these various project teams against the master plan.

Then part of it entails the political place of getting people to agree that the current situation is really kind of sub-optimal, and that there's a future state that's really desirable - and really identifying the hindering factors. Usually people, process and cultural norms in terms of moving from a current state to a future state. Is that a fair characterization of a change agent? LP: Yes. I think it is. I think you're exactly on track when you're saying they've got to have the ability to put together a master plan. They have to also be good at communicating it and socializing it. The people at the minimum level maybe are not. They may be really good data people, but they may not be good change agents.

It's also imperative that the better and best marketing operations people figure out how to improve peoples' skill sets, so they feel they can be successful. This process and the analytics can be overwhelming and potentially scary. And some people on the team may not have all the skills they need to do it and be successful. That can be very demoralizing. So a good marketing operations person/team makes sure that those skills are brought up to speed in a positive way, so that people can feel they can be successful.

MM: One of the things I've also found in successful change facilitators or change agents is that they know when to bring in what kind of external consultant or subject-matter expert to get a very specific piece of work done of the overall master plan.

LP: Yes. I agree with you that they recognize when the time is right to have other people from outside on the team. Not only outside of the company, but sometimes outside of the organization.

MM:Yes. Perfect.

And that leads into the next part you were talking about - that this person should really have an understanding of how to research the requirements - both business requirements and technical requirements - for a new system. As well as being able to correlate those business and technical requirements to existing or needed infrastructure investments. 
LP: This would be talking about the "better." Right?

MM:Yes. And specifically, how to integrate those systems and data flows within the existing infrastructure and other kind of enterprise systems that are part of that whole business ecosystem.

LP: Yes. Exactly. So the people at the beginning level probably don't have those skills.

MM: That's fair enough.

LP: They may not even need them.

MM: Yes.

LP: But if you want to really become more proficient, and this is your vision of the ideal life for you, then you've got to have these capabilities.

MM: In many respects, Laura, what we're really doing here is describing your ideal customer.

LP: We certainly are.

MM: We're also then in the course of identifying an ideal client, outlying a basic career track. These are the kinds of things that somebody who wants to bring performance-driven or performancebased marketing into an organization... These are the kinds of things that you'll probably need to have under your belt.

LP: I think so. What do you think, Michael? MM: I agree. That's why I'm spending a little more time here, kind of developing out or drilling into some of the skills portfolio of a successful director or vice-president of marketing operations.

The next thing that you said was that they have this ability to take data and form them as insights and communicate them up the channel and down into the organization.

LP: And across. Like with sales, for example. Across as opposed to up. Right? Or product people. Some organizations' product is separate from the marketing organization.

MM: This communication in an interaction ultimately undergirds or frames the emergence of metrics that then perhaps later show up in the dashboards.

LP: Absolutely.

MM: So part of the development of the dashboard is really a collaborative effort across an organization from this change leader who says, "Here are some insights. What are the best ways of tracking material changes in how our marketing organization adds value to the company?"
LP: We agree with you. It is a collaborative effort. That's why when we do this work with companies, generally the people engaged in the process - the initiatives - both the development and its adoption - are not just inside marketing. Sales, finance, product and customer service - people are all part of the development. You want the rest of the organization to recognize marketing's value. You want to be able to show them that marketing is measuring the things the business cares about and is able to relate the effort back to the business impact.

MM: Then this leads into the whole notion of business intelligence. So in terms of our good better best, we might be punching out of the "better" and into the "best." But this executive understands the notion of or the underlying principles of "business intelligence," and understands the difference between standard reporting, descriptive modeling, maybe even "predictive modeling" and using scorecards and benchmarks as data frames for really assembling data from multiple sources, and conditioning it into high-level insights.

LP: Yes.

MM: And at "best," we'll be able to take those data and start doing models and simulations that allow them to essentially predict future outcomes, based on certain investments and certain configurations of resources.

LP: Exactly. And at a minimum, at that "best" level, they can at least do scenario analysis. MM: Are there particular tools that this particular person would use at the level of scenario modeling and predictive models? LP:Yes, but because we are tool agnostic, I want to be careful about endorsing anything in particular. But firms such as SAS, Business Objects, Cognos, Unica, Alterian, Aprimo, etc may be a good place to start.

MM: Sure.

LP: There may also be tools in other parts of the business that the marketing organization can leverage.

MM: Fair enough.

MM: Are there any other types of niche tools you've seen your clients use to really do that? LP: No. Many of our customers are just at the early stages of tools, bringing on things such as Alterian, Eloqua and/or Aprimo for example. MM: Fair enough. 
In terms of some of the dashboards that you've seen clients develop and start to use are there any particular systems or tools that you've seen your clients use in creating these dashboards?

LP: Many of them start with the tools we've already mentioned because many of these products generate various reports. And these are what the company is familiar with. The challenge is that these reports are often at the campaign level. To get to the next level they may try to use Siebel or Sales Force but these have their limitations as well. In fact, every tool does; they are limited by the data that are put in. To create an outcome-based dashboard, we find that a lot of companies need to start almost at a manual level. Outcome-based dashboards are transformative. So by going through the manual process, we can find out whether the data are available and where. And if not, what data will need to be collected and where it will be stored and how. Once the metrics catalog is created, the organization can begin to think about tools.

So the very first step is to validate the linkages between the needles that you're moving. You want to be able to identify data that will help you assess whether the input/activity produced the objective and outcome. In the beginning this may need to be done manually. Once the linkages have been validated, the data defined and the process created, then you can look at how to bring it all together. Whether that's through SAP or Oracle or SAS or any other system/tool.

MM: Great.

I want to thank you very much for a really great interview today. LP: Michael, thank you. 